Untersuchung einiger aus der Hippursäure entstehenden Producte;

von Nicolaus Socoloff und Adolph Strecker.

Es giebt wenige organische Säuren, über deren rationelle Zusammensetzung die Ansichten der Chemiker so getheilt sind, als über die der Hippursäure. Die Hippursäure ist nämlich eine Säure von ziemlich complexer Zusammensetzung, und erleidet durch Einwirkung verschiedener Reagentien mannichfaltige Zersetzungen, deren jede zur Annahme einer besonderen Gruppirung der Elemente führte. Fehling schlors aus der Zersetzung der Hippursäure durch Bleihyperoxyd, wodurch sie in Benzamid nnd Kohlensäure verwandelt wurde, dafs sie Benzamid als näheren Bestandtheil enthalte, verbunden mit einer Säure, welche durch Aufnahme von Sauerstoff in Kohlensäure übergehe.

$\mathrm{C}_{14} \mathrm{H}_{7} \mathrm{NO}_{2}$ Benzamid,

\begin{tabular}{cccc}
$\mathrm{C}_{4}$ & $\mathrm{H}_{2}$ & $\mathrm{O}_{4}$ & Fumarsäure, \\
\hline $\mathrm{C}_{18}$ & $\mathrm{H}_{9}$ & $\mathrm{NO}_{6}$ & Hippursäure.
\end{tabular}

Da übrigens die Fumarsäure beim Kochen mit Bleihyperoxyd und Wasser nicht verändert wird, so entspricht obige Zusammensetzung nicht vollkommen der Spaltung, aus welcher sie abgeleitet wurde.

Pelouze betrachtete die Hippursäure zusammengesetzt aus:

\begin{tabular}{llllll}
$\mathrm{C}_{14}$ & $\mathrm{H}_{6}$ & $\mathrm{O}_{2}$ & Bittermandelöl, \\
$\mathrm{C}_{2}$ & $\mathrm{H}$ & $\mathrm{N}$ & & Blausäure, \\
$\mathrm{C}_{2}$ & $\mathrm{H}_{2}$ & $\mathrm{O}_{4}$ & Ameisensäure, \\
\hline $\mathrm{C}_{18}$ & $\mathrm{H}_{9}$ & $\mathrm{NO}_{0}$ & Hippursãure.
\end{tabular}

Diese Ansicht stützt sich besonders auf das Auftrelen von Blausäure bei der trocknen Destillation der Hippursäure, so wie auf die Entstehung von Benzoësäure, Kohlensäure und Ammoniak bei der Behandlung der Hippursäure mit Braunstein und verdünnter Schwefelsäure. Da übrigens die mit Aldehyden gepaarten 
Ameisensäuren (Milchsäure, Mandelsäure u. s. w.) unler denselben Umständen die Aldehyde im freien Zustande abgeben, so läfst sich auch die von Pelouze angegebene Conslitulion der Hippursäure nicht mit der Zersetzung dieser Säure in Uebereinstimmung bringen.

Nachdem Dessaignes gefunden halte, dafs durch die Einwirkung der concentrirten und kochenden Säuren und Alkalien die Hippursäure in Benzoësäure und Glycocoll zerfalle, neigte sich die Mebrzahl der Chemiker der Ansicht zu, diese Säure als eine gepaarte Verbindúng von Benzoësäure und Glycocoll zu betrachten. Aber dann kann man, wie Berzelius richtig bemerkt, die Metamorphose nicht verstehen, welche das Bleihyperoxyd bewirkt.

Diese verschiedenen Ansichten über die Constitution der Hippursäure setzen demnach in dieser Säure eine Atomgruppe voraus, welche 14 Aeq. Kohlenstoff enthält, und zwar eine Benzoylverbindung, sowie eine zweite Gruppe, welche 4 Aeq. Kohlenstoff einschliefst und unter Umständen wieder in zwei andere Körper, von je 2 Aeq. Kohlenstoff, zerlegt werden kann. Sie unterscheiden sich hauptsächlich dadurch, dafs sie den Slickstoff entweder mit der Benzoylgruppe, oder mit der zweiten Gruppe verbunden annehmen.

Da es jedoch je nach der auf die Hippursäure stattfindenden Einwirkung gelingt, den Stickstoff auf die eine oder die andere der beiden Atomgruppen zu übertragen, so scheint es dem Verhalten der Säure entsprechender, den Stickstoff nicht in näherer Verbindung mit einer einzelnen der beiden Atomgruppen, sondern mit der durch die Vereinigung derselben entstandenen gepaarten Verbindung anzunehmen, und hiernach die Hippursäure als ein Amid der gepaarten Säure $\mathrm{C}_{18} \quad \mathrm{H}_{8} \mathrm{O}_{8} \mathrm{zu}$ betrachten. Zur Isolirung dieser Säure liels sich nicht die Einwirkung der Alkalien oder Säuren auf die Amidsäure anwenden, weil die gepaarte Säure selbst hierdurch eine Zersetzung erleidet, aber 
es war zu erwarten, dafs die salpetrige Säure, in ähnlicher Weise wie aus anderen Amiden, die stickstoffrreie Säure abscheide. Der Versuch hat, wie einer von uns schon fruher*) miltheilte, in der That der Voraussetzung entsprochen, indem es ihm gelang, die in der Hippursäure vorausgreselzte Atomgruppe $\mathrm{C}_{18} \mathrm{H}_{8} \mathrm{O}_{8}$ für sich darzustellen. Wir haben diese Säure, welche wir Benzoglycolsäure nennen, einer näheren Untersuchung unterworfen, und theilen die Resultate derselben im Folgenden mit.

Die Hippursäure stellten wir aus Pferdeharn nach der elwas abgeänderten Methode von Gregory dar; wie Städeler **) mit Recht bemerkt, bewirkt das Kochen des Harns mit Kalkmilch nur wenig Vortheil, da bei dem Abdampfen der stark alkalischen Flüssigkeit braune harzartige Körper entstehen, welche die Reinigung der Hippursäure erschweren. Wir haben daher nach Greg or y's Vorschlag den Harn zuerst mil Kalkmilch kurze Zeit sieden lassen, und ihn hierauf colirt, sodann aber nicht unmittelbar abgedampft, sondern erst sorgfältig mit Salzsäure neutralisirt und kochend eingeengt. Die auf Zusatz von Salzsäure aus der eingeengten und erkalteten Flüssigkeit abgeschiedene Hippursäure war, nach dem Abwaschen mit kaltem Wasser, fast ganz weils und zeigte nur einen schwachen Stich in's Röthliche. Wir haben diese Säure, ohne sie weiter zu reinigen, zu folgenden Versuchen angewendet, da wir uns überzeugt haben, dafs die daraus entstehende Benzoglycolsäure weit leichter vollkommen farblos zu erhalten ist, als die Hippursäure.

Bringt man zu einer wässerigen Lösung von salpetrigsaurem Kali Hippursäure, so bemerkt man keine Veränderung; selbst beim Kochen tritt keine Zersetzung ein. Die Hippursäure ist eine schwächere Süure als die salpetrige Säure, und treibt diese aus ihren Salzen nicht aus. Ebensowenig findet eine Einwir-

*) Diese Annalen LXVIII, 54.

**) Ebendaselbst LXXVII, 18. 
kung stalt, wenn man Hippursäure in concentrirter Schwefelsäure löst, und aus einer Mischung von Stärkmehl und Salpetersäure entwickelte salpetrige Säure einleitet.

Leítet man dagegen durch eine wässerige Lösung von Hippursäure, in welcher man gepulverte Hippursäure vertheilt hat, einen Strom von salpetriger Säure, so findet zwar in der Kälte keine, oder nur eine äufserst geringe Einwirkung statt, aber in der Wärme wird die Hippursäure unter Entwickelung von Stickstoff zersetzt. Da übrigens ein grolser Theil der salpetrigen Säure unter diesen Umständen in Salpetersäure und Stickstoftoxyd sich zerlegt, welche erstere auf die neue Säure in der Hitze eine Zersetzung ausiibt, so haben wir auch diesen Weg verlassen und sind zu folgender, ursprünglich von uns angewendeten Methode zurückgekehrt, welche die besten Resultate lieferte.

Die lufttrockene Hippursäure wurde zerrieben und nach und nach, unter fortwährendem Reiben in einem Mörser, mit soviel käuflicher Salpetersäure versetzt, dafs ein dünner Brei entstand. Die Masse brachten wir in einen hohen Glascylinder (um eine grö(sere Flüssigkeitssäule zu erhalten) und leiteten einen Strom von Stickstoffoxydgas (aus Kupfer mit Salpetersäure entwickelt) durch die Lösung. Der Cylinder darf hierbei höchstens bis zur Hälfte mit der Masse angefüllt seyn, da ein starkes Aufschäumen eintritt, welches leicht einen Verlust veranlassen könnte. Findet die Entwickelung des Stickstoffoxydgases nicht zu lebhaft statt, so wird es vollständig absorbirt, so dafs keine rothen Dämpfe über der Flüssigkeit sich bilden, was bei einem lebhafteren Strom kaum zu vermeiden ist. Die gröfseren Blasen, welche in die Flüssigkeit eintreten, verschwinden, und aus der Flüssigkeit entwickelt sich eine Menge kleiner Gasbläschen, welche reines Stickstoffgas sind. Bei jedem Umschütteln der Flüssigkeit wird diese Entwickelung bedeutend verstärkt. Die anfangs in der Flüssigkeit nur suspendirte Hippursäure ver- 
schwindet allmälig; sic löst sich auf und die Flüssigkeil wird ganz klar. Das Ende der Zerselzung laifst sich nicht leicht erkemnen, und wir haben daher stets so lange Stickstofloxyd eingeleitet, bis dic Lösung eine deullich grüne Farbe angenommen hatte; ein Uebcrschufs von Stickstoffoxyd schadet nicht, sondern wirkt, indem eine gewisse Menge des Lösungsmittels dadurch weggenommen wird, vortheilhaft. Die ganze Operation ist in 5 bis 6 Stunden vollendel; wir haben dabei nie erhitzt, da wir befürchtelen, dals dic neugebildete Säure eine weitere Zersetzung crleiden möchte.

Schon während des Durchleitens von Slickstoffoxydgas scheidet sich aus der klaren Lösung ein Theil der aus der Hippursäure entstandenen Benzoglycolsäure ab; die Hauplmasse fälı aber erst auf Zusalz von Wasser nicder. Man vermischt daher die Lïsung in Salpelersäure mit viel Wasser, läfst die hicrbei warm gewordene Flïssigkeit sich wicder ablïhlen, und filtrirt die abgeschiedene Benzoglycolsäure durch cin an der Spitze doppelt gelegles Papiertilter. Die Säure wurde auf dem Filter mit möglichst kaltem Wasser so lange ausgewaschen, bis das. Waschwasser bei der Prüfung mit Eisenvitriol und Schwefelsäure keine Reaction auf Salpetersäurc mehr zeigle. Dieses Auswaschen dauert, wegen der kürnig krystallinischen Beschaffenheil der Masse, gar nicht lange, und verursacht daher auch keinen bedeutenden Verlust an Säure.

Dic auf diese Weise dargestellte rolıe Benzoglycolstiure ist schwach gelblich gefärbl und bedarf cincr weiteren Reinigung. Wir haben sic in Wasser vertheilt mit Kalkmilch neutralisirt, wobei der anfangs dünne Brei nach lurzer Zcit fast ganz zu ciner fusten Masse gestand. Obwohl das Kalksalz leichter löslich ist als dic freie Säure, so scheint es doch wegen seiner voluminösen Beschaffenheit auf den ersten Anblick umgekchrt zu seyn. Das abgeschiedene Kalksalz wurde durch Erwärmen wieder in der Flüssigkeit gelöst, und schied sich aus der filtrirten 
Lösung nach dem Erkalten in sehr langen und feinen Nadeln aus. Das Salz enthält sehr viel Mutterlauge aufgesogen und erscheint daher gelblich gefärbt, wird aber durch Abwaschen mit wenig kaltem Wasser und starkes Auspressen vollkommen weifs orhalten. Die Multerlauge liefert beim weiteren Verdampfen neue Krystallisationen desselben Kalksalzes. Die letzten Mullerlaugen liefern ein gelb gefärbtes Salz, welches sich nur durch Behandlung mit Thierkohle farblos erhalten lärst, und gewöhnlich neben benzoglycolsaurem Kalk etwas benzoësauren Kalk enthält.

Die mit Wasser verdünnte Salpetersäure hält noch eine gewisse Menge von Benzoglycolsäure in Lösung; wir haben letztere daraus gewonnen, indem wir die Säure mit kohlensaurem Kali genau neutralisirten, kochend einengten und von dem beim Erkalten anschiefsenden Salpeter die Flüssigkeit abgossen, diese wieder weiter einengten und die letzte Multerlauge, welche die ganze Menge des sehr löslichen benzoglycolsauren Kali's gelöst enthält, mit concentrirter Salpetersäure versetzten. Die hierdurch abgeschiedenen Krystalle enthalten gewöhnlich neben Benzoglycolsäure ziemlich viel Benzoësäure, welche letztere wir in folgender Weise entfernten. Die eine Hälfte des Gemenges wurde mit Kalk genau neutralisirt, hierauf die zweite Hälfte zugefügt und die Mischung zur 'Trockne eingedampft. Da die Benzoglycolsäure eine stärkere Säure ist als die Benzoësäure, so war in der Mischung letztere Säure frei, erstere zum grölsten Theil mil Kalk verbunden enthalten. Wir behandelten daher den Rückstand in Payen's Extractionsapparat mit Aether und entfernten hierdurch die Benzoësäure vollständig. Das ungelöst zurückgebliebene Kalksalz war vollkommen weifs und rein; die ätherische Lösung enthielt die ganze Menge der Benzoësäure und etwas Benzoglycolsäure.

Die Darstellung der Benzoglycolsäure in reinem Zustande aus dem Kalksalz ist äufserst einfach. Das Kalksalz wurde in Wasser gelöst und in der Kälte mit Salzsäure versetzt, wobei 
die Benzoglycolsäure als leichtes, krystallinisches, vollkommen weilses Pulver sich abscheidet. In gröfseren Krystallen wurde die Benzoglycolsäure aus ihrem Kalksalz dargestellt, indem dieses in Alkohol gelöst und mit Schwefelsäure versetzt wurde; die von dem niederfallenden Gyps abfiltrirte Flüssigkeit schied beim freiwilligen Verdunsten die Säure in ziemlich grofsen, farblosen Prismen ab. Dic Krystalle haben das Ansehen von Prismen mit $37^{\circ} 40^{\prime}$ und $142^{\circ} 20^{\prime}$ Kanten, welche durch überwiegende Ausdehnung zweier Prismaflächen in Form dünner Tafeln erscheinen. Genauere Bestimmungen anderer Flächen und des Systems liefsen sich bei der grolsen Dünne der Krystalle nicht ausführen.

Die aus der alkoholischen Lösung abgeschiedenen Krystalle wurden der Analyse unterworfen.

Bei $100^{\circ}$ verloren sie nicht an Gewicht, schienen aber bei längerem Verweilen in dieser Temperalur, namentlich bei feuchter Luft, eine allmälige Zersetzung zu erleiden.

I. 0,4165 Grm. bei $100^{\circ}$ getrockneter Benzoglycolsäure gaben beim Verbrennen mit chromsaurem Bleioxyd 0,9205 Grm. Kohlensäure und 0,1795 Grm. Wasser.

II. 0,3532 Grm. lufttrockene Substanz gaben, mit Kupferoxyd und überchlorsaurem Kali verbrannt, 0,7758 Grm. Kohlensäure und 0,1441 Grm. Wasser.

In 100 Theilen :

I. $\quad$ I.

$\begin{array}{lrr}\text { Kohlenstoff } & 60,28 & 59,91 \\ \text { Wasserstoff } & 4,79 & 4,53 .\end{array}$

Diese Zahlen führen, bei Zugrundelegung des aus den Salzen abgeleiteten Aequivalentes zu der Formel :

$$
\begin{array}{llll}
\mathrm{C}_{18} & \mathrm{H}_{8} & \mathrm{O}_{8},
\end{array}
$$

welche sich in folgender Weise mit dem Mittel der Analysen vergleicht : 


\begin{tabular}{cllrrr} 
& & & \multicolumn{2}{c}{ berechnet } & Nittel der Versuche \\
\cline { 3 - 5 } 8 & Aeq. & Kohlenstoff & 108 & 60,00 & 60,09 \\
8 & Wasserstoff & 8 & 4,44 & 4,66 \\
8 & Sauerstoff & 64 & 35,56 & 35,25 \\
\hline 1 Aeq. & Benzoglycolsäure & 180 & 100,00 & $100,00$.
\end{tabular}

Die Benzoglycolsäure ist in kaltem Wasser sehr schwer löslich, leichter in heifsem Wasser, wodurch sie allmälig zersetzt wird. Erhitzt man sie mit einer zur Lösung ungenügenden Menge von Wasser, so schmilzt sie zu ölartigen Tropfen. In Alkohol und in Aether ist sie leicht löslich. Die Benzoglycolsäure schmilzt beim Erhitzen auf dem Platinblech und erstarrt beim Erkalten wieder krystallinisch. Beim stärkeren Erhitzen liefert sie zum Husten reizende Dämpfe, worunter Benzoësäure leicht bemerkt wird, und hinterläfst einen geringen kohligen Rückstand, der beim Glühen leicht verbrennt.

\section{Benzoglycolsaure Salze.}

Die Salze der Benzoglycolsäure sind in Wasser grölstentheils löslich, und viele von ibnen lösen sich auch in Alkobol. Sie besitzen eine neutrale Reaction und einen wenig auffallenden, eigenthümlichen Geschmack. Aus der wässerigen Lösung der meisten Salze scheiden slärkere Säuren, selbst Essigsäure, die Benzoglycolsäure in kleinen Krystallen ab.

Benzoglycolsaures Kali. Wir haben dieses Salz durch genaue Sältigung der Säure mit kohlensaurem Kali, oder durch Zersetzung des Kalksalzes mit kohlensaurem Kali dargestellt. Das Salz ist in Wasser und in Alkohol sehr leicht löslich, und krystallisirt nur schwer und undeutlich. Beim Erkalten der heifsgesättigten Lösung scheidet es sich in aufserordentlich dünnen, sehr breiten Tafeln ab. Beim freiwilligen Verdunsten hinterbleibt es in blumenkohlartigen Massen.

Benzoglycolsaures Natron. Dieses Salz krystallisirt viel leichter als das vorhergehende, und man erhält es beim Erkalten 
der heifsgesättigten Lösung in ziemlich grofsen, rhombischen 'Tafeln. Bei $100^{\circ}$ wird das Salz undurchsichlig, bleibt aber glänzend.

I. 0,5656 Grm. der lufttrockenen Substanz verloren bei $100^{\circ}$ $0,1188 \mathrm{Grm}$. Wasser und gaben 0,1572 Grm. schwefelsaures Natron.

II. $0,5332 \mathrm{Grm}$. lufttrockenes Salz verloren bei $100^{\circ} 0,1124$ Grm. Wasser.

In 100 Theilen :

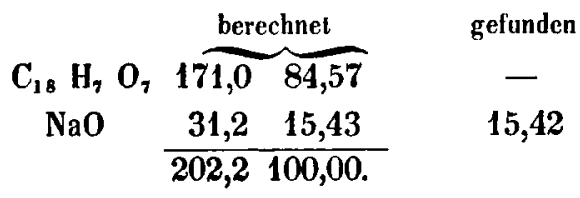

Die Menge des Krystallwassers entspricht 6 Aequivalenten :

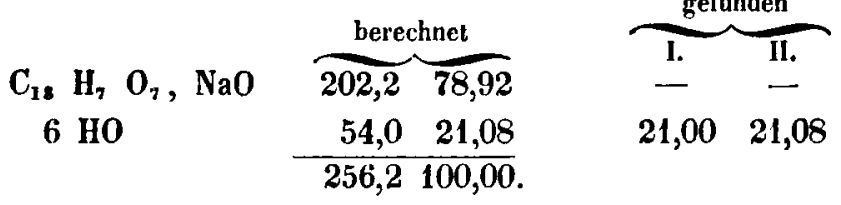

Das Ammoniaksalz wurde auf dieselbe Weise wie das Kalisalz dargestellt. Es verliert beim Abdampfen Ammoniak.

Benzoglycolsaurer Kalk. Dieses Salz wurde, wie früher erwähnt, durch Sättigen der freien Säure mit Kalkmilch dargestellt. Es besitzt im höchslen Grade die Eigenschaft, übersättigte Lösungen zu bilden, so dals zuweilen ganz kalte Lösungen, aus welchen selbst ein Theil schon auskrystallisirt ist, beim Coliren durch ein feines 'Tuch sich trüben und nach einigen Augenblicken vollständig unter Ausscheidung des sehr voluminösen Kalksalzes zu einer dicken Gallerte erstarren. Filtrirt man diese ab, so erstarrt die Multerlauge gewöhnlich nach kurzer Zeit abermals. Das Salz bildet feine, wavellitartig gruppirte, seidenglänzende Nadeln. Die Krystalle verändern sich nicht an der Luft und verlieren bei $100^{\circ}$ nicht an Gewicht. 
1. $0,3900 \mathrm{Grm}$. bei $100^{\circ}$ getrockneter Substanz gaben, mit chromsaurem Bleioxyd verbrannt, 0,744 Grm. Kohlensäure und 0,146 Grm. Wasser.

II. $0,8270 \mathrm{Grm}$. bei $100^{\circ}$ getrockneter Substanz gaben 0,2702 Grm. schwefelsauren Kalk.

III. 0,4634 Grm. lufttrockene Substanz gaben 0,1488 Grm. schwefelsauren Kalk.

In 100 Theilen :

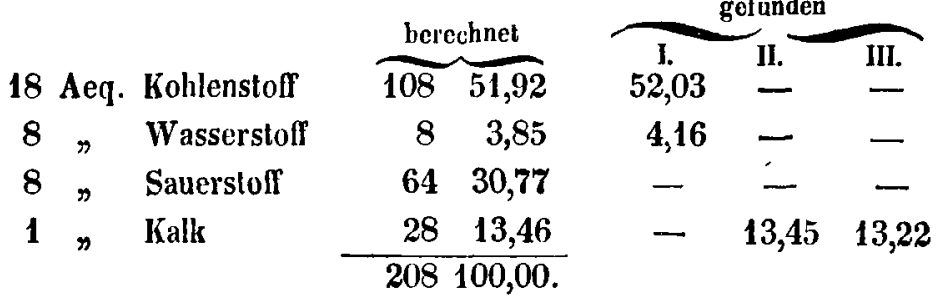

Das Salz hält also bei $100^{\circ}$ I Aeq. Krystallwasser zurück, und seine Formel mufs

$$
\mathrm{CaO} \cdot \mathrm{C}_{18} \mathrm{H}_{7} \mathrm{O}_{7}+\mathrm{HO}
$$

geschrieben werden.

Dieses 1 Aequivalent Kryslallwasser geht erst bei $120^{\circ} \mathrm{C}$. fort, was folgende Analyse zeigt.

$0,4474 \mathrm{Grm}$. bei $100^{\circ}$ getrockneter Substanz verloren bei $120^{\circ} 0,0192 \mathrm{Grm}$. Wasser und gaben $0,146 \mathrm{Grm}$. schwefelsauren Kalk.

In 100 Theilen :

$$
\begin{array}{ccccc}
\mathrm{C}_{18} \quad \mathrm{H}_{7} & \mathrm{O}_{\gamma} & \overbrace{171}^{\text {berechnet }} & 85,93
\end{array} \quad \begin{gathered}
\text { gefunden } \\
\mathrm{CaO}
\end{gathered}
$$

Das krystallisirte Salz enthält :

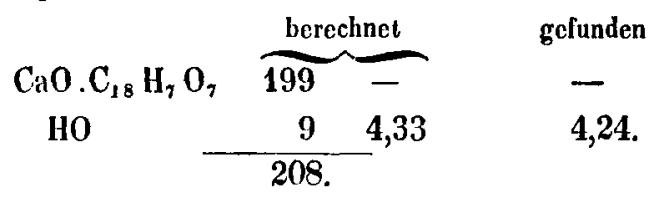


Die Löslichkeil des Salzes ergiebt sich aus folgenden Versuchen :

36,8924 Grm. der bei $11^{\circ}$ gesältigten Lösung gaben 0,2910 Grm. schwefelsauren Kalk.

22,5443 Grm. kochender Lösung (der Siedepunct liegt bei $101^{\circ}, 5$ C.) gaben $0,8998 \mathrm{Grm}$. schwefelsauren Kalk.

100 Thle. Wasser von $11^{\circ}$ lösen also 2,36 Thle. Salz, oder 1 Thl. Salz löst sich in 42,32 Thln. Wasser.

Bei dem Kochen lösen dagegen 100 Thle. Wasser 13,26 Thle. Salz, oder 1 Thl. Kalksalz löst sich in 7,54 Thln. Wasser.

Benzoglycolsaurer Baryt. Dieses Salz krystallisirt, ähnlich wie das Kalksalz, in feinen, seidenglänzenden Nadeln. Es enthält 2 Aeq. Krystallwasser und verliert diese bei $100^{\circ}$.

1) $0,4424 \mathrm{Grm}$. Iufttrocknes Salz verloren bei $100^{\circ} 0,0295$ Grm. Wasser und gaben 0,1944 Grm. schwefelsauren Baryt.

2) $0,5088 \mathrm{Grm}$. lufttrocknes Salz verloren bei $100^{\circ} 0,0342$ Grm. Wasser und gaben 0,2232 Grm. schwefelsauren Baryt.

\begin{tabular}{|c|c|c|c|c|}
\hline \multirow{4}{*}{$\begin{array}{c}\mathrm{C}_{18} \mathrm{H}_{7} \mathrm{O} \\
\mathrm{BaO}\end{array}$} & \multirow{2}{*}{\multicolumn{2}{|c|}{ berechnet }} & \multicolumn{2}{|c|}{ gefunden } \\
\hline & & & 1. & 2. \\
\hline & 171 & 69,06 & - & 一 \\
\hline & 76,6 & 30,94 & 30,93 & 30,90 \\
\hline & 247,6 & $100,00$. & & \\
\hline
\end{tabular}

Das krystallisirte Salz enthält :

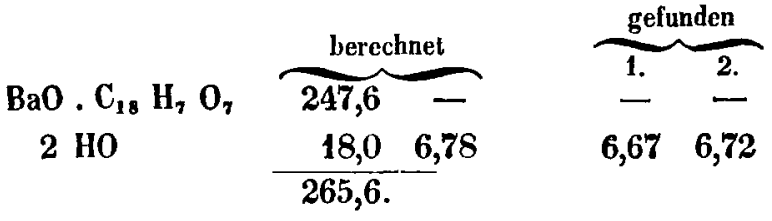

Benzoglycolsaure Magnesia. Wir haben dieses Salz durch Zusammenbringen der kochenden Lösungen von benzoglycolsaurem Kalk und schwefelsaurer Magnesia und Ausziehen der beim Erkalten erstarrten Masse mit absolutem Alkohol dargestellt. Das Salz krystallisirt beim Abdampfen und Erkalten in 
langen, aufserordentlich feinen und zarten Nadeln und zeigt daher cine sehr voluminöse Beschaffenheit.

Benzoglycolsaures Eisenoxyd. Versctzl man eine Lösungr von benzoglycolsaurem Kalk mit Eisenchlorid, so entsteht ein voluminöser, unkrystallinischer Niederschlag von fleischrolher Farbe, der bei dem Trocknen an der Luft oberflächlich dunkler wird. Das Salz ist in Wasser vollkommen unlöslich, und läfsł sich daher so lange auswaschen, bis das Waschwasser mit Blullaugensalz keine Färbung melır zcigt.

$0,6238 \mathrm{Grm}$. der lufttrockenen Substanz verloren bei $100^{\circ}$ 0,1692 Grm. Wasser und gaben 0,1058 Grm. Eisenoxyd. Das bei $100^{\circ}$ getrocknete Salz besilzt hiernach die Formel

$$
2 \mathrm{Fe}_{2} \mathrm{O}_{3} .3\left(\mathrm{C}_{18} \mathrm{H}_{7} \mathrm{O}_{7}\right) \text {. }
$$

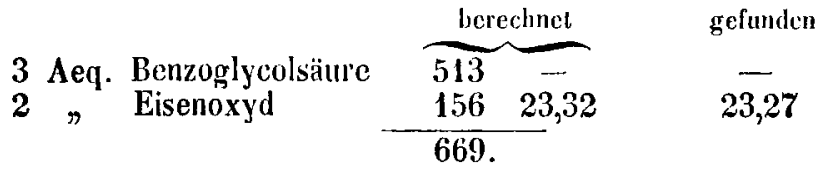

Das lufttrockene Salz verlor bei $100^{\circ} 27,12$ pC. Wasser, entsprechend 28 Ae(j. Wasser $(27,36$ pC.).

Benzoglycolsaures Zinkoxyd. Es wurde durch Zusanmenbringen der kochenden Lösung des Kalksalzes mit Chlorzink beim Erkalten in farblosen, langen, dïnnen Nadeln crloalten, welche sich sternförmig gruppirten und das ganze Gofäfs crfiillten. Das Salz wurde abfiltrirt, mit kallem Wasser elwas abgewaschen und zwischen Fliefspapier ausgeprelst. Das zur Analyse verwendete Salz wurde nochmals umkrystallisirt.

I. $0,4861 \mathrm{Grm}$. luftrockenen Salzes verloren bei $100^{\circ} 0,0721$

Grm. Wasser und gaben 0,0805 Grm. Zinkoxyd.

II. $0,5200 \mathrm{Grm}$. desselben Salzes verloren bei $100^{\circ} 0,0758$ Grm. Wasser und gaben 0,0854 Grm. Zinkoxyd.

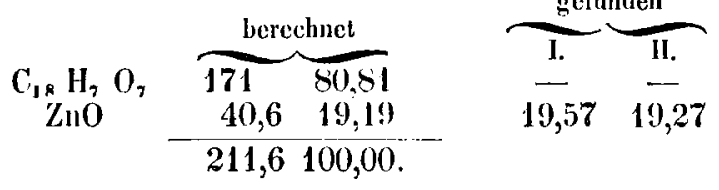


Das kryslallisirle Salz entlıält :

\begin{tabular}{|c|c|c|c|c|}
\hline \multirow{4}{*}{$\begin{array}{l}\mathrm{ZnO}, \mathrm{C}_{18} \mathrm{H}_{7} \mathrm{O}_{7} \\
4 \mathrm{HO}\end{array}$} & \multicolumn{2}{|c|}{ lerechnet } & \multicolumn{2}{|c|}{ gefunden } \\
\hline & & & I. & II. \\
\hline & 211,6 & 85,46 & - & - \\
\hline & 36,0 & 14,54 & 14,83 & 14,58 \\
\hline & 247,6 & 100,00 . & & \\
\hline
\end{tabular}

Benzoglycolsaures Kupferoxyd. Wir stellten dieses Salz aus dem Kíalksalz durch Vermischen der kochenden Lösung desselben mit salpetersaurem Kupferoxyd dar, wobei es sich nach dem Erkalten in reichlicher Menge abscheidet. Es krystallisirt in schün blauen, rhombischen Tafeln. Die Krystalle wurden abfiltrirt, mil kaltem Wasser abgewaschen und umkrystallisirt. Es löst sich sehr schwer in kaltem Wasser, so dafs die erkaltete Lösung kaum blau gefärbt erscheint; in heifsem Wasser ist es etwas leichter löslich. Erwärmt man die Krystalle mit einer zur völligen Auflösung unzureichenden Menge von Wasser, so bildet das Ungelüsle ein grünes Pulves, wahrscheinlich wasserfreies Salz. Bei $100^{\circ}$ werden die Krystalle gamz grün und undurchsichlig, ohne dafs die Flächen ihren Glanz verlieren.

Benzoglycolsaures Bleioxyd. Die kalle Lösung des benzoglycolsauren Kalks wird durch neutrales essigsaures Bleioxyd reichlich in käisigen Flocken gefüllt. Der Niederschlag löst sich schwer in kaltem Wasser auf, beim Erhitzen mit Wasser schmilzt er zuerst, löst sich hierauf vollständig auf und scheidet sich bei dem Erkalten wieder ab. Der crste Absalz ist amorph, nach dem Erkalten scheidet sich das Bleisalz in Krystallen aus. Wir haben zur Darstellung des Salzes für die Analyse den ursprünglichen käsigen Niederschlag in vielem kaltem Wasser beinahe vollständig aufgelöst, abfiltrirt, und die Lösung der freiwilligen Verdunslung überlassen. Nach einigen Tagen hatte sich das Bleisalz in Krystallen abgeschieden, welche sich zu dichten, halbluggelfürmigen Massen vereinigt hatten. Dieses 
Salz schmilzt bei $100^{\circ}$ vollständig, auch nachdem es das Krystallwasser verloren hat.

I. 0,5308 Grm. des lufttrocknen Salzes verloren bei $100^{\circ}$ $0,0208 \mathrm{Grm}$. Wasser und gaben 0,342 Grm. schwefelsaures Bleioxyd.

II. $0,4282 \mathrm{Grm}$. verloren bei $100^{\circ} 0,0172 \mathrm{Grm}$. Wasser und gaben 0,2742 Grm. schwefelsaures Bleioxyd.

Diesen Analysen zufolge ist dieses Salz eines der häufigen 3 basischen Bleisalze :

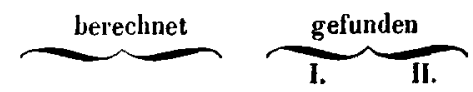

2 Aeq. Benzoglycolsäure $342 \quad 50,44$

3 , Bleioxyd

\begin{tabular}{rr}
336 & 49,56 \\
\hline 678 & 100,00
\end{tabular} 49,$41 \quad 49,16$

Der gefundene Wassergehalt entspricht der Formel :

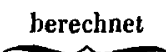

$3 \mathrm{PbO}, 2\left(\begin{array}{lll}\mathrm{C}_{18} & \left.\mathrm{H}_{7} \mathrm{O}_{2}\right) & 678\end{array}\right.$ $+3 \mathrm{HO}$

$$
\frac{27}{705} \quad 3,83 \quad 3,92 \quad 4,03
$$

Nach längerem Stehen halten sich aus der Flüssigkeit, aus welcher das vorhergehende Salz auskrystallisirt war, dünne und kurze, zarte Nadeln abgeschieden, welche wie die meisten anderen Salze sternförmig gruppirt waren. Diese Krystalle liefsen sich leicht durch Schlämmen von dem vorhergehenden Salz trennen; sie wurden auf dem Filter gesammelt, mit kaltem Wasser abgewaschen und zwischen Fliefspapier geprelst.

Beim Erhitzen auf $100^{\circ}$ schmilzt das Salz theilweise und erleidet allmälig eine Zersetzung. Bei einem Versuche slieg der Gehalt an Bleioxyd bis $41,8 \mathrm{pC}$.

0,2642 Grm des luftrockenen Salzes gaben 0,1408 Grm. schwefelsaures Bleioxyd. 
Dieses Salz ist einfach-benzoglycolsaures Bleioxyd :

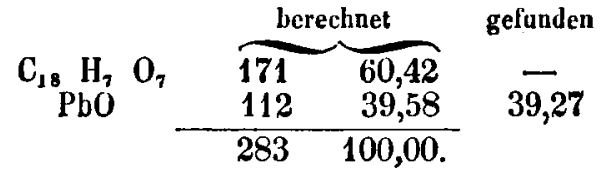

Vermischt man eine kochende Lösung von benzoglycolsaurem Kalk mit neutralem essigsaurem Bleioxyd, so entsteht ein amorpher weilser Niederschlag, der sich beinahe vollständig in Essigsäure löst. Er ist ein Gemenge basischer Salze, welches bei einer Probe 78,6 pC. Bleioxyd enthielt.

Fällt man die kalte Lösung von benzoglycolsaurem Kalk mit basisch essigsaurem Bleioxyd, so entsteht ein flockiger Niederschlag, der beim Kochen in Wasser nicht schmilzt, und sich nur sehr wenig in Wasser löst. Der etwas ausgewaschene Niederschlag wurde mit kaltem Wasser übergossen, die Lösung abfiltrirt und stehen gelassen. Nach mehreren Tagen hatten sich dem neutralen Bleisalz ähnlich sehende Krystalle abgeschieden, welche durch Schlämmen von etwas beigemengtem kohlensaurem Bleioxyd getrennt wurden.

$0,2132 \mathrm{Grm}$. der lufttrockenen Substanz verloren bei $100^{\circ}$ $0,0056 \mathrm{Grm}$. Wasser und gaben 0,2246 Grm. schwefelsaures Bleioxyd.

Diefs entspricht der Formel :

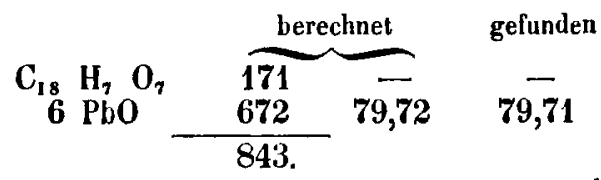

Der Verlust an Wasser entspricht der Formel :

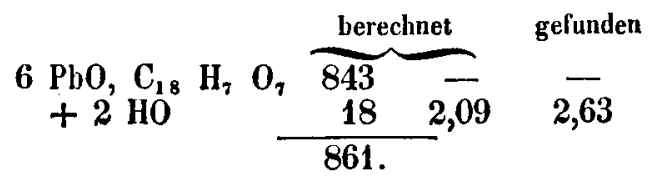

Die durch directe Fällung erhaltenen Bleiniederschläge sind stets Gemenge verschiedener basischer Bleisalze. 
Benzoglycolsaures Silberoxyd. Wir stellten dieses Salz durch Fällen einer neutralen Lösung von benzoglycolsaurem Ammoniak mit salpetersaurem Silberoxyd dar. Der Niederschlag wurde mit etwas kaltem Wasser ausgewaschen und aus kochendem Wasser umkrystallisirt. Er stellt feine, weilse, mikroscopische Krystalle dar, welche sich in feuchlem Zustande sehr leicht am Licht schwärzen. Bei künstlicher Beleuchtung dargestellt, war das Salz vollkommen weifs. In kaltem Wasser ist es schwer löslich, ziemlich leicht in kochendem.

Das lufttrockene Salz ist wasserfrei ${ }_{s}$ und verlor bei $100^{\circ}$ nur $0,1 \mathrm{pC}$. an Gewicht.

I. 0,3308 Grm. des bei $100^{\circ}$ getrockneten Salzes gaben, mit chromsaurem Bleioxyd verbrannt, 0,458 Grm. Kohlensäure und $0,0808 \mathrm{Grm}$. Wasser.

II. 0,433 Grm. desselben Salzes gaben 0,1628 Grm. Silber. III. 0,576 Grm. einer anderen Bereitung gaben 0,2170 Grm. Silber.

Diese Analysen führen $2 \mathrm{u}$ der Formel $\mathrm{AgO}, \mathrm{C}_{18} \quad \mathrm{H}_{2} \mathrm{O}_{3}$, wie folgende Zusammenstellung zeigt :

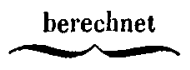

18 Aeq. Kohlenstoff
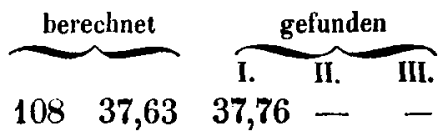

$7 \gg$ Wasserstoff

7 , Sauersioff

$7 \quad 2,44$

$37,76--$

\begin{tabular}{lrr}
$1 》$ Silberoxyd & 116 & 40,42 \\
\hline 1, benzoglycols. Silberoxyd & 287 & $100,00$.
\end{tabular}

$56 \quad 19,51$

$2,71--$

1

$-40,3840,48$

Benzoglycolsäure-Aether. Schon bei längerem Stehen der alkoholischen Lösung der Benzoglycolsäure entwickelt sich ein eigenthümlicher Geruch, welchen wir der Bildung einer zusammengesetzten Aetherart zuschreiben zu dürfen glaubten. Wir versuchten die Darstellung derselben durch Einleiten von trockener Salzsäure in eine alkoholische Lösung von benzoglycolsaurem Kalk, worin noch mehr Kalksalz vertheilt war. 
Auf Zusalz von Wasser schied sich eine ölartige Flüssigkeit ab, welche aber, wie die Analyse zeigte, fast nur Benzoëäther war. Die Benzoglycolsäure hatle durch Einwirkung der Salzsäure daher eine Zersetzung erlitten.

Aus der vorhergehenden Beschreibung der benzoglycolsauren Salze ergiebt sich, dals die Benzoglycolsäure HO, $\mathrm{C}_{\mathrm{i}} \mathrm{H}_{7} \mathrm{O}, 1$ Aeq. durch Metalloxyde vertretbares Wasser enthält, und mithin ebensoviel Basis sältigt, als die Hippursäure, aus welcher sie entstanden ist.

Die Entstehung der Säure aus Hippursäure wird durch die Gleichung :

$$
\underbrace{\mathrm{C}_{18} \mathrm{H}_{0} \mathrm{NO}_{0}}_{\text {Hippursäure. }}+\mathrm{NO}_{3}=\underbrace{\mathrm{C}_{18} \mathrm{H}_{8} \mathrm{O}_{8}}_{\begin{array}{c}
\text { Benzoglycol- } \\
\text { säure. }
\end{array}}+\mathrm{HO}+2 \mathrm{~N}
$$

dargestellt, und ist demnach der Bildung anderer Säuren aus ihren Amiden vollkommen entsprechend. Wir finden aber hier ein von dem gewöhnlich beobachteten abweichendes Verhältnifs, indem in den übrigen Fällen die Amidsäure 1 Aeq. Basis weniger sälligt, als die freie Säure. Diese Thatsache steht übrigens nicht ganz isolirt da. Die Asparaginsäure $\mathrm{C}_{8} \mathrm{H}_{7} \mathrm{NO}_{8}$ sältigt, wie fruhere Versuche Liebig's, welche neuerdings durch Wolff*) bestätigt wurden, zeigten, ebensoviel Basis, als die daraus durch eine der obigen ähnliche Reaction entstehende Aepfelsäure, und ein ähnliches Verhältnifs findet zwischen der Salicylsäure und dem Amid derselben statt.

Dieses Sätligungsverhältnifs zeigt einen wesentlichen Unterschied zwischen den gewöhnlichen Amiden, Amidsäuren und den dieser Gruppe nahestehenden Körpern, wie Hippursäure, Alanin, Anthranilsäure u. s. w. an, obwohl beide gegen salpetrige Säure sich in der nämlichen Weise verhalten.

-) Dieso Annalen Bd. LXXV, S. 296. 
Die Zusammensetzung der benzoglycolsauren Salze weicht in Beziehung auf den Kohlenstoff-, Wasserstof - und Basisgehalt nur wenig von der Zusammenselzung der hippursauren Salze $a b$, wie z. B. folgende Zusammenstellung der Zusammenselzung des benzoglycolsauren Kalks und des hippursauren Kalks zeigt.

Benzoglycolsaurer Kalk

$\begin{array}{lll}\mathrm{C}_{18} & 108 & 54,27\end{array}$

\begin{tabular}{lrr}
$\mathrm{H}_{7}$ & 7 & 3,52 \\
$\mathrm{O}_{7}$ & 56 & 28,14 \\
$\mathrm{CaO}$ & 28 & 14,07 \\
\hline & 199 & 100,00
\end{tabular}

Hippursaurer Kalk

$\mathrm{C}_{18} \quad 108 \quad 54,55$

H. 84,04

N $14 \quad 7,07$

Os $40 \quad 20,20$

\begin{tabular}{rrr}
$\mathrm{CaO}$ & 28 & 14,14 \\
\hline 198 & $100,00$.
\end{tabular}

Wir haben uns daher stets durch Prüfung mit Kalium von der Abwesenheit von Stickstoff in unseren Salzen überzeugt. Nur das neulrale Bleisalz, welches aus einer nicht ganz reinen Säure dargeslellt worden war, gab Spuren von Slickstoff hierbei zu erkennen.

Zersetzungsproducte. Die Untersuchung der Zersetzungsproducte der Benzoglycolsäure gab uns Aufschlufs über die Constitulion dieser Säure.

Wie früher erwähnt wurde, erleidet die Benzoglycolsäure schon beim Kochen der wässerigen Lösung der freien Süure eine allmälige Zersetzung; in ihren Salzen hat sie indessen eine grölsere Beständigkeit, so dafs man diese lange Zeit im Sieden erhalten kann, ohne dafs eine Zersetzung sich beınerken läfst. Weit rascher als durch blolses Wasser wird die Benzoglycolsäure beim Erwärmen mit verdünnten Säuren zersetzt; mit den Wasserdämpfen verflüchligt sich hierbei Benzoësäure, während eine andere Säure in der zurückbleibenden Flüssigkeil gelöst enthalten ist. Um die einzelnen Producle näher zu untersuchen, verfulren wir in folgender Weise.

Wir kochten Benzoglycolsäure mit Wasser unter Zusalz einer kleinen Menge verdünnter Schwefelsäure einige Tage lang auf dem Sandbad, und ersetzlen das verdampfte Wusser 
von Zeit zu Zeil. Die Flüssigkeit stöfst hierbei sehr stark und selbst das Einbringen von Platinblech hinderte das Stofsen nicht vollständig. Die Hauptmasse der Säure ist nach einigen Stunden zersetzt; da aber eine Beimengung von etwas unzersetzter Benzoglycolsãure der weiteren Reindarstellung der Producte sehr hinderlich ist; da man ferner kein Mittel hat, den Zeitpunkt leicht zu erkennen, in welchem die Zerselzung vollendet ist: so setzten wir das Kochen einige Tage lang fort. Mit den Wasserdämpfen verflüchligt sich eine grofse Menge von Benzoësäure; durch Einengen auf ein kleines Volum und Erkaltenlassen schieden wir den gröfsten Theil der in der Lösung noch enthaltenen Säure ab. Obwohl die beim Erkalten sich abscheidenden Krystaltblütter ihren physikalischen und chemischen Eigenschaften nach als reine Benzoësäure sich zu erkennen gaben, so zogen wir doch vor, diefs durch die Analyse festzustellen.

Die Krystalle wurden mil kaltem Wasser etwas abgewaschen, mit Kalkmilch neutralisirt und das sich ausscheidende Kalksalz der Analyse unterworfen.

$0,513 \mathrm{Grm}$. des bei $100^{\circ}$ gelrockneten Kalksalzes gaben 0,2454 Grm. schwefelsauren Kalk.

Wir stellen dieses Resullat neben die Zusammenselzung des benzoësauren Kalks :

$$
\begin{array}{lcc}
\mathrm{C}_{14} \mathrm{H}_{5} \mathrm{O}_{3} & \begin{array}{c}
\text { berechnet } \\
80,14
\end{array} & \text { gefunden } \\
\mathrm{CaO} & 19,86 & \\
\cline { 2 - 2 } & & 19,70 \\
& 100,00 &
\end{array}
$$

Die Mutterlauge, ans welcher die Benzoësäure auskrystallisirt war, wurde mit kohlensaurem Baryt neutralisirt, von dem niederfallenden schwefelsauren Baryl ahfiltrirt und stark eingedampft. Beim Erkalten krystallisirte anfangs Nichts aus, und erst nach dem Abdampfen zur Syrupconsistenz schied sich beim ruhigen Stehen nach einigen 'Tagen das Barytsak einer, neuen Säure in weifsen, harten, krystallinischen Krusten ab. Wir 
gossen die Multerlauge von den Krystallen ab, und entfernten durch Pressen zwischen Fliefspapier die Flüssigkeit möglichst vollständig. Ein Auswaschen war bei der grofsen Löslichkeit dieses Salzes in Wasser nicht möglich.

Das Barytsalz schmilzt beim Erhitzen zu einer vollkommen klaren Flüssigkeil, die bei dem Erkalten wieder krystallinisch erstarrt. Bei stärkerem Erhitzen bläht es sich bedeutend auf und hinterlä[st unter Verbreitung eines eigenlhümlichen Geruchs kaum grau gefärbten kohlensauren Baryt, der sich sehr rasch weils brennt.

Die Analyse dieses Salzes ergab folgende Resultate :

I. 0,4890 Grm. lufttrockener Substanz verloren bei $100^{\circ}$ $0,0016 \mathrm{Grm}$. Wasser und gaben 0,3306 Grm. kohlensauren Baryt.

II. $0,4785 \mathrm{Grm}$. luftrockner Substanz verloren bei $100^{\circ}$ Nichts an Gewicht und gaben $0,3246 \mathrm{Grm}$. kohlensauren Baryt.

III. 0,5021 Grm. derselben Substanz gaben beim Verbrennen mit chromsaurem Bleioxyd 0,3096 Grm. Kohlensäure und $0,1028 \mathrm{Grm}$. Wasser.

Diese Zahlen führen zu der Formel :

$$
\mathrm{BaO}, \mathrm{C}_{4} \mathrm{H}_{3} \mathrm{O}_{5} \text {, }
$$

wie folgende Zusammenstellung zeigt :

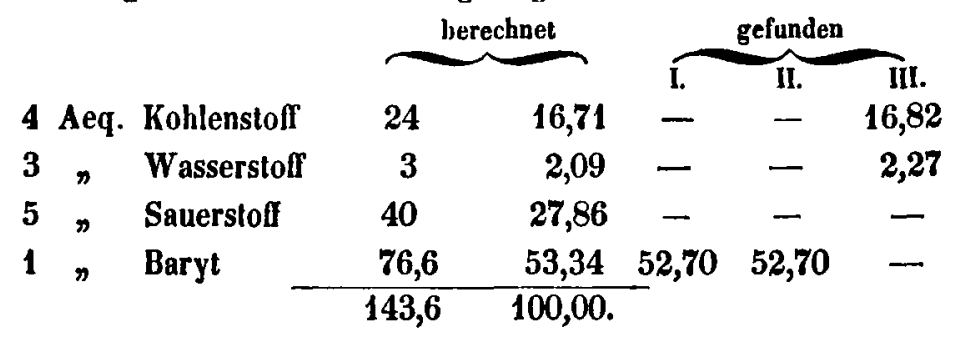

Der geringe Unterschied der berechneten und der gefundenen Zahlen rührt von einer kleinen Beimengung von benzoësaurem Baryt her, wie leicht aus der Darstellung zu entnehmen 
ist. In der Multerlauge, welche wir mit kohlensaurem Baryt neutralisirten, mufste eine der geringen Löslichkeit der Benzoësäure entsprecbende Menge dieser Säure gelöst bleiben, welche mit Baryt neutralisirt sich dem anderen Barylsalze beimengte, wodurch der Barytgehalt desselben erniedrigt, der Koblenstoffund Wassersloffgehalt etwas erhöht wurde.

Die in dem Barytsalz enthaltene Säure $\mathrm{C}_{4} \mathrm{H}_{3} \mathrm{O}_{5}$ oder als Säurehydrat gedacht $\mathrm{C}_{4} \quad \mathrm{H}_{4} \mathrm{O}_{6}$ kommt mit der Säure überein, als deren Amidverbindung man das Glycocoll belrachten kann, und welche daher von Laurent ${ }^{*}$ ) den Namen Glycolsäure erhalten hat. Es ist nämlich :

$$
\underbrace{\mathrm{C}_{4} \mathrm{H}_{4} \mathrm{O}_{6}}_{\text {Glycolsäure. }}+\mathrm{NH}_{3}=\underbrace{\mathrm{C}_{4} \mathrm{H}_{5} \mathrm{NO}_{4}}_{\text {Glycocoll. }}+2 \mathrm{HO} .
$$

Laurent hat diese Säure nicht dargestellt, er, sowie Gerhârdt, glaubten aber, eine von Horsford durch Oxydation des Glycocolls erhaltene Säure, für deren Barytverbindung Horsford aus seinen Analysen die Formel : $\mathrm{BaO}, \mathrm{C}_{3} \mathrm{H}_{3} \mathrm{O}_{3}$ abgeleitet halle, für Glycolsäure $\mathrm{C}_{4} \mathrm{H}_{4} \mathrm{O}_{6}$ ansehen zu dürfen, indem sie dem von Horsford analysirten Barylsalz die Formel $\mathrm{BaO}, \mathrm{C}_{4} \mathrm{H}_{3} \mathrm{O}_{3}$ gaben. Sie nahmen zu diesem $Z$ wecke an, bei der Verbrennung des Salzes sey 1 Aeq. Kohlensäure bei dem Baryt geblieben, dessen Kohlenstoffgehalt sie dem gefundenen Kohlenstoff zurechneten. Da aber Horsford, wie er selbst sagt, mit chromsaurem Bleioxyd verbrannte, wobei bekanntlich alle Kohlensäure sich verflüchtigt, so läfst sich die Umrechnung nicht rechtferligen, un so weniger, als auch der gefundene Barylgehalt mit der neuen Formel nicht übereinstimml. Dafs die von Horsford erhaltene Säure von unserer Glycolsäure verschieden ist, ergiebt sich schon daraus, dafs Horsford aus einer ammoniakalischen Lösung seiner Säure das Barylsalz durch

7) Ann. de Phys. et do Chim. [3] XXIII, 112. 
Zusalz von Chlorbarium niederschlug, während unsere Gycolsäure selbst in der concentrirteslen Lösung auf Zusalz von Chlorbarium keinen Niederschlag giebt.

Wenn wir aber doch den Namen Glycolsäure beibehalten, so geschieht diefs, weil, wie wir sogleich zeigen werden, unsere Säure zu Glycocoll in der Beziehung steht, welche Laurent zur Aufstellung der Bezeichnung Glycolsäure veranlafste.

\section{Glycolsäure.}

Zur Darstellung der Glycolsäure aus dem Barylsalz verselzten wir die wässerige Lösung desselben mit verdünnter Schwefelsäure, so lange noch ein Niederschlag von schwefelsaurem Baryt entstand, filtrirten ab, und dampften das Filtrat im Wasserbade ein. Der syrupartige Rückstand löste sich in Aether völlig auf, und die ätherische Lösung hinterliefs beim Verdunsten im Wasserbad die Glycolsäure wieder in Gestalt eines dünnen Syrups, welcher bei längerem Stehen nicht krystallisirte.

Die Glycolsäure ist eine etwas dicke Flüssigkeit, welche sich mit Wasser, Alkohol oder Aether in jedem Verhältnifs mischl. Sie besitzt einen stark sauren Geschmack und giebt mit keinem Metallsalze einen Niederschlag. Wie man siebt, zeigt die Glycolsäure in ihren Eigenschaften die grörste Aehnlichkeit mit der Milchsäure; wir haben nur Eine Reaction auffinden können, wodurch beide Säuren sich von einander unterscheiden lassen. Verselzl man eine Lösung von Glycolsäure mit essigsaurem Bleioxyd und fügt einen Ueberschufs von Ammoniak zu, so entsteht ein weilser flockiger Niederschlag, während bei Anwendung von Milchsäure unter denselben Verhältnissen die Lösung klar bleibt.

\section{Glycolsaures Zinkoxyd.}

Zur Darstellung dieses Salzes wurde die Glycolsäure mit 
Wasser verdünnt und mit überschüssigem kohlensaurem Zinkoxyd erhilzl; die Lösung des Zinksalzes wurde abfiltrirt und eingeengt. Nach dem Erkalten batten sich krystallinische Krusten abgeschieden, welche die gröfste Aehnlichkeit mit dem auf ähnliche Weise dargestellten milchsauren Zinkoxyd besitzen. Die einzelnen Krystalle sind kleine, farblose und durchsichtige Säulen, welche sternförmig um mehrere Punkte sich gruppiren.

Die Analyse des Salzes ergab folgende Resultate :

I. $\mathbf{0 , 4 3 7} \mathrm{Grm}$. bei $100^{\circ}$ gelrockneter Substanz gaben, mit chromsaurem Bleioxyd verbrannt, 0,3436 Grm. Kohlensäure und $0,118 \mathrm{Grm}$. Wasser.

11. 0,2974 Grm. desselben Salzes gaben 0,1118 Grm. Zinkoxyd. Diese Zahlen führen zu der Formel :

$$
\mathrm{ZnO}, \mathrm{C}_{4} \mathrm{H}_{3} \mathrm{O}_{5} \text {, }
$$

wie folgende Vergleichung der berechneten und gefundenen Zahlen zeigt :

4 Aeq. Kohlenstoff

3 , Wasserstoff

5 , Sauerstoff

1 Zinkoxyd

1 )

\begin{tabular}{crc}
\multicolumn{2}{c}{ berechnet } & gefunden \\
3 & 22,33 & 22,69 \\
40 & 2,79 & 3,00 \\
40,5 & 37,21 & - \\
107,67 & 37,64 \\
$100,00$. &
\end{tabular}

Die Krystalle verăndern sich an trockner Luft nicht, bei $100^{\circ}$ werden sie undurchsichlig, unter Verlust von Wasser.

I. 0,3484 Grm. luftrockenes glycolsaures Zinkoxyd verloren bei $100^{\circ} 0,051$ Grm. Wasser.

II. $0,5178 \mathrm{Grm}$. desselben Salzes verloren bei $100^{\circ} 0,0744$ Grm. Wasser.

Dieser Wasserverlust entspricht 2 Aeq. Wasser :

$$
\text { berechnet }
$$

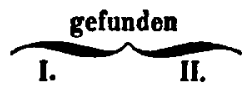

$\mathrm{ZnO}, \mathrm{C}_{4} \mathrm{H}_{3} \mathrm{O}_{5} \quad 85,66$

$2 \mathrm{HO}$

$14,34 \quad 14,64 \quad 14,37$. 
Das Zinksalz ist in Alkohol unlöslich, in kaltem Wasser löst es sich schwer, leichter in heilsem.

32,762 Grm. bei $20^{\circ} \mathrm{C}$. gesältigter Lösung des Zinksalzes gahen $0,962 \mathrm{Grm}$. bei $100^{\circ}$ getrockneten Rückstand.

100 Theile der Lösung enthalten also 2,94 Theile wasserfreies glycolsaures Zinkoxyd, oder 1 Theil Zinksalz löst sich in 33 Theilen Wasser auf.

\section{Glycolsaures Siberoxyd.}

Wir versuchten die Darstellung dieses Salzes durch Zersetzung des glycolsauren Baryts mit schwefelsaurem Silberoxyd. Beim Abdampfen der hierdurch erhaltenen Lösung fand aber eine Zersetzung stalt, und es schieden sich schwarze Flocken ab. Selbst beim Verdunsten der Lösung über Schwefelsäure im luftleeren Raum fand eine allmälige Schwärzung statt, und der hinterbleibende zähe Rückstand löste sich nicht mehr vollständig in Wasser auf. Ebenso wenig gelang es uns, aus der wässerigen Lösung auf Zusatz von Alkohol das glycolsaure Silberoxyd zu fällen, und selbst ein Zusatz von Aether verursachle keine Trübung; die durch Zersetzung des Barytsalzes erballene Lösung des Silbersalzes war vermuthlich zu verdünnt.

Die Glycolsäure $\mathrm{C}_{4} \mathrm{H}_{4} \mathrm{O}_{6}$ besitzl, wie die vorher angeführten Versuche zeigen, die gröfste Aehnlichkeit mit der ibr homologen Milchsäure $\mathrm{C}_{8} \mathrm{H}_{6} \mathrm{O}_{6}$; die Menge, welche durch die Formel $\mathrm{C}_{4} \quad \mathrm{H}_{4} \mathrm{O}_{6}$ dargestellt ist, neutralisirt 1 Aeq. Basis (nicht 2 Aeq., wie Laurent und Gerhardt ihrer Theorie nach annehmen). Wir müssen es aber dahin gestellt sein lassen, ob die Glycolsäure eine zweibasische Säure ist, in welchem Falle ihre Formel verdoppelt und $2 \mathrm{HO}, \mathrm{C}_{8} \mathrm{H}_{6} \mathrm{O}_{20}$ geschrieben werden mürste. Die Analyse des glycolsauren Zinkoxyds zeigt, dafs dasselbe entsprechend dem paramilchsauren Zinkoxyd (Milchsäure aus der Fleischflüssigkeit) 2 Aeq. Krystallwasser 
enthält. Leider besaisen wir zu wenig Material, um die Verwandlungen dieser Säure weiler zu untersuchen. Es wäre insbesondere interessant gewesen, das Verhalten der Glycolsäure in der Wärme zu untersuchen, da vermuthlich dieselbe, ähnlich wie die Milchsäure, wasserfrei zu erhalten ist. Wenn wir die Milchsäure als eine gepaarte Verbindung von Ameisensäure mit dem Aldehyd der Essigsäure betrachten dürfen; so wäre die Glycolsäure eine entsprechende Verbindung mit dem Aldehyd der Ameisensäure :

$$
\begin{aligned}
& \underbrace{\mathrm{C}_{6} \mathrm{H}_{6} \mathrm{O}_{6}}_{\text {Milchaäure }}=\underbrace{\mathrm{C}_{2} \mathrm{H}_{2} \mathrm{O}_{4}}_{\text {Ameisensăure }}+\underbrace{\mathrm{C}_{4} \mathrm{H}_{4} \mathrm{O}_{2}}_{\text {Aldehyd. }} \\
& \underbrace{\mathrm{C}_{4} \mathrm{H}_{4} \mathrm{O}_{6}}_{\text {Glycolsăure }}=\underbrace{\mathrm{C}_{2} \mathrm{H}_{2} \mathrm{O}_{4}}_{\text {Ameisensäure }}+\underbrace{\mathrm{C}_{2} \mathrm{H}_{2} \mathrm{O}_{2}}_{\text {Formylaldehyd. }}
\end{aligned}
$$

Wahrscheinlich wird der Formyl-Aldehyd bei der trocknen Destillation der Glycolsäure, oder bei der Behandlung derselben mit Oxydationsmitteln erhalten. Bei der Behandlung der Hippursäure mit Bleihyperoxyd entwickelt sich bekanntlich ein äufserst stechender Geruch, und Schwarz beobachtete, dals das wässerige Destillat die Reaclion der Aldehyde gegen Silberlösung zeigle. Es scheint uns sehr wahrscheinlich, dafs diese Erscheinungen durch den Aldehyd der Ameisensäure verursacht sind, welcher, weil er bei gewöhnlicher Temperatur gasförmig ist, bis jelzt der Beobachtung entgangen ist.

Die Glycolsäure entsteht auch durch Einwirkung von salpetriger Saure auf Glycocoll. Das Glycocoll wird schon in wässeriger Lösung durch salpetrige Säure unter Entwickelung von Stickstoff zersetzt. Dampft man die Lösung hierauf ein, so findet durch die aus der salpetrigen Säure entstandene Salpetersäure eine Oxydation der Glycolsäure stalt und man erhält im Rückstand Krystalle von Oxalsäure. Schüttelt man dagegen die mit salpetriger Säure behandelte Glycocolllösung mit Aether, so entzieht dieser die Glycolsäure der wässerigen Lösung und 
beim Verdunsten des Aethers hinlerbleibt die Glycolsäure in Gestalt einer syrupdicken Flüssigkeit, welche die Eigenschaften der aus der Benzoglycolsäure erhaltenen Säure zeigte. Da übrigens die Darstellung der Glycolsäure aus letzlerer Säure einfacher ist, so haben wir es stets vorgezogen, sie auf diesem Wege darzustellen. Die Entstehung der Glycolsüure aus dem Glycocoll wird durch die Gleichung :

$$
\underbrace{\mathrm{C}_{4} \mathrm{H}_{3} \mathrm{NO}_{4}}_{\text {Glycocoll }}+\mathrm{NO}_{3}=\underbrace{\mathrm{C}_{4} \mathrm{H}_{4} \mathrm{O}_{6}}_{\text {Glycolsăure }}+\mathrm{HO}+2 \mathrm{~N}
$$

dargestellt.

Man gelangt daher zu denselben Resultaten, wenn man die Hippursäure zuerst mit salpelriger Säure behandelt und die Benzoglycolsäure hierauf durch Kochen mit Säuren zerlegl, oder wenn man zuerst die Hippursäure in Benzoësäure und Glycocoll zerlegt, und letzleres hierauf mit salpetriger Säure behandelt.

Nach dem Vorhergehenden zerfällt also die Benzoglycolsäure leicht in Benzoësäure und Glycolsäure, und wir haben Gründe, zu glauben, dafs dieselbe aus diesen ihren Bestandtheilen wieder hergestellt werden kann, was nach folgender Glcichung vor sich gehen murs :

$$
\underbrace{\mathrm{C}_{14} \mathrm{H}_{6} \mathrm{O}_{4}}_{\text {Benzoèsäure }}+\underbrace{\mathrm{C}_{4} \mathrm{H}_{4} \mathrm{O}_{6}}_{\text {Glycolsäure }}=\underbrace{\mathrm{C}_{18} \mathrm{H}_{8} \mathrm{O}_{8}}_{\text {Benzoglycolsäure. }}+2 \mathrm{HO} \text {. }
$$

Mangel an Glycolsäure verhinderte den Versuch zur Besläligung dieser Ansicht, aber wir haben denselben mit der mit itmr homologen Milchsäure gemacht. Wir erhitzten nämlich zu diesem Zwecke ein Gemenge von Milchsäure und Benzoësäure auf $180^{\circ}$ und erhielten diese Temperatur, so lange noch eine Entwickelung von Wasserdampf sich beobachten liefs. Der Rückstend erstarte beim Erkalten zu einer harzartigen Masse, welche in Wasser unter Zusalz von Kali gelöst wurde. Zu der warmen Lösung fügten wir so lange verdünnte Schwefelsäure, als beim Erkalten noch eine Krystallisation von Benzoësäurc sich beob- 
achlen liels, und bis die sich abscheidenden Krystalle eine andere Form zeigten. Bei diesem Puncte wurde die abgeschiedene Säure abfiltrirt und das Filtrat in der Kälte mit verdünnter Schwefelsäure versetzt. Es entstand ein reichlicher Niederschlag, der beim Kochen mit Wasser zuerst schmolz, in vielem Wasser sich auflöste und beim Erkalten zum Theil in Tropfen, zum Theil in Krystallen sich ausschied. Diese Säure wurde in Aether gelöst und durch Verdunsten wieder krystallisirt erhalten; wir neutralisirten sie mit Ammoniak und fällten mit Silberlösung. Der Niederschlag wurde, ohne ihn umzukrystallisiren, analysirt.

$0,508 \mathrm{Grm}$. des bei $100^{\circ}$ getrockneten Salzes gaben 0,1878 Grm. Silber oder 39,7 pC. Silberoxyd.

Diefs kommt der Formel AgO. $\mathrm{C}_{20} \mathrm{H}_{9} \mathrm{O}_{7}$ sehr nahe, welche 38,5 pC. Silberoxyd verlangt.

Das benzoësaure Silberoxyd enthält über $50 \mathrm{pC}$. Silberoxyd und es war nach unserer flüchtigen Darstellung nicht zu vermeiden, dals sich der neuen Säure elwas Benzoësäure beimengen mulste. Die Bildung dieser neuen Säure, welche wir Benzomilchsäure nennen wollen, erklärt sich leicht nach der Gleichung :

$$
\underbrace{\mathrm{C}_{14} \mathrm{H}_{6} \mathrm{O}_{4}}_{\text {Benzoëbăure }}+\underbrace{\mathrm{C}_{6} \mathrm{H}_{6} \mathrm{O}_{6}}_{\text {Milchaäure }}=\underbrace{\mathrm{C}_{20} \mathrm{H}_{10} \mathrm{O}_{8}}_{\text {Benzomilchsăure. }}+2 \mathrm{HO}
$$

Wir behalten uns weitere Angaben uber diese Säure vor, und werden zugleich untersuchen, ob die Milchsäure mit anderen organischen Säuren entsprechende gepaarte Verbindungen bildet. 